\title{
Face-to-face discussions: networking or opinions exchange?
}

\author{
Simone Righi ${ }^{1}$ and Timoteo Carletti $^{2}$ \\ 1 Department of Economics, CRED and naXys, University of Namur (FUNDP). Mailing address: \\ Department of Economics, 8 Rempart de la Vierge, 5000 Namur, Belgium. simone.righi@fundp.ac.be \\ 2 Department of Mathematics and naXys, University of Namur (FUNDP). Mailing address: Department \\ of Mathematics, 8 Rempart de la Vierge, 5000 Namur, Belgium. timoteo.carletti@fundp.ac.be
}

\begin{abstract}
We use recent results of [4] on face-to-face contact durations to try to answer the question: why do people engage in face-to-face discussions? In particular we focus on behavior of scientists in academic conferences. We show evidence that macroscopic measured data are compatible with two different micro-founded models of social interaction. We find that the first model, in which discussions are performed with the aim of introducing oneself (networking), explains the data when the group exhibits few well reputed scientists. On the contrary, when the reputation hierarchy is not strong, a model where agents' encounters are aimed at exchanging opinions explains the data better.
\end{abstract}

Keywords: face-to-face discussion, opinion dynamics, social interactions

Mathematical physics is today a well accepted framework where model and study social phenomena. After an initial phase relying mostly on qualitative models $[5,8$, $15]$, scientists begun to obtain quantitative results $[1,6,7,14]$, mainly using quite abstract models able to capture universal patterns in human behavior without carefully adding complicating details that could blind a complete understanding of the relationships between the assumptions done and the outcomes observed.

Thanks to the advancements in technology a new phase recently started, where researchers have access to fine measurements about real world interactions. The huge amount of data (internet based applications, mobile network [2,3,9-11], faceto-face interactions [4]) is however often associated with a lack of a mathematical foundation of the emergent properties observed. Moreover not everything can be measured; understand why do people "internally" act in such a way as to determine the measured global properties, would necessitate a hierarchical model, whose levels 
will range from the "brain level", though the "individual level" to eventually arrive to the "group level". The complexity of such model would be too important to allow any useful analysis.

In the present paper we make a step forward and "measure the unmeasurable" using such kind of data. More precisely, we use recent results of face-to-face contact durations (see for instance [4]) to try to answer the question: why do people engage face-to-face discussions? While the present paper focus on interactions among scientists in conferences and workshops, cases to which data from [4] referred to, our setup is flexible enough to be applied to other situations and datasets.

Starting from a simple microscopic model we show evidence that macroscopic measured data, notably supra linear growth of total discussion time with respect to the agent connectivity, are compatible with two different scenarios based on the group structure. These can be summarized in two micro-founded models of social interaction. In the first one, we assume discussions among the agents to be performed about a neutral topic and thus the opinions exchanges do not influence the discussion times nor the selection of the partner, i.e. the goal of the discussions is to introduce himself to the partner (networking). On the other hand, in the second model we assume that agents' encounters are aimed to find a compromise among the subject of discussion and thus there could be an opinion update as consequence of the encounters.

We also assume that, in both models, each agent is characterized by a publicly known and accepted level of reputation and that agents aim to engage discussions with highly reputed peers. In the case of a scientific meeting this could be the $h$-factor of the individuals.

We find that when the group exhibits clearly identified well reputed people, then the outcomes of our model are consistent with the assumption that discussions are engaged mainly to share time with the most reputed person, namely agents perform networking actions, i.e. the first scenario does apply. On the other hand, when 
the reputation hierarchy is not strong, our findings are consistent with the hypothesis that people's discussions are finalized to opinions exchange, namely the second scenario holds.

We are aware that our models are very simple ones and that more variable could affect the discussion times among agents. However, as already stated, our goal is to reduce as much as possible the number of free parameters and thus, for instance, associate possible causes of long/short discussions to the experimental data.

The homogeneity assumption of the agent behavior, is also a strong one, thus in a second part of the paper we will relax this hypothesis by allowing the group to be composed by agents aimed at exchanging opinions and also agents doing networking. We will be thus interested in studying the robustness of our results as a fraction of the relative fraction of agents.

The paper is organized as follows. Section 1 will present the two main models, while Section 2 is aimed at reporting some numerical results. Finally Section 3 will contain the results of the non homogeneous model.

\section{The Models}

\section{$1.1 \quad$ Networking (Model A)}

To test the hypothesis that agents could be motivated, in their interactions, by the simple willingness to do networking, defined as introducing oneself and spending time to exchange personal informations with the more reputed persons, without the need of exchange valuable opinion, we introduce the following model (hereby named Model A).

Consider a closed group of $N$ fixed agents, where social interactions last for discretized blocks of $\tau$ seconds; in the following we chose a value of $\tau=20$ seconds to obtain simulations with the same time resolution of the experimental data from Barrat et al. [4]. As already stated each agent $i$ is characterized by a level of reputation $h_{i}$, hereby defined by a positive real number distributed according to a power law 
distribution $\frac{1}{h^{\gamma h}}$, where $\gamma_{h}$ is a parameter of the model. As the dynamics unfolds, free agents ${ }^{3}$ search for a partner to initiate a discussion. Our working assumption is that agents have a preference to engage in discussions with peers with high reputation, that is a publicly known value. Indeed, the probability of selecting an agent as partner is proportional to his reputation, properly renormalized. Namely the probability to select agent $j$ is proportional to $h_{j} / \sum_{k=1}^{N} h_{k}$.

When a free agent meets another currently idle agent, the couple engages in a discussion for a time $\Delta t$, extracted from the power law distribution $\frac{1}{(\Delta t)^{\gamma}}$, where the parameter $\gamma$ has been fixed to the value 1.5 that can be extracted from the experimental data of Barrat and al. [4]. However, the partner selection rule does not guarantee that each attempt will be successful, i.e. the wanted partner will be free at that time, in this case the first agent needs to wait for $\Delta t_{w}$ seconds before trying to initiate another conversation. The idea is to mimic the fact that an agent lose some time after an unfruitful approach by looking around or just taking a cup of coffee. We assume the distribution of the waiting times to be again a power law [16], $\frac{1}{\left(\Delta t_{w}\right)^{\gamma w}}$. We assume moreover that the exponent $\gamma_{w}$ varies for each agent and it is influenced by the degree of success of past interactions, more precisely $\gamma_{w}=1+x_{i}$, where $x_{i}$ indicates the degree of frustration incurred, by the $i$-th agent that promotes the conversation, as a consequence of past interactions:

$$
x_{i}=\frac{\text { Number of successful interactions of the } i \text {-th agent }}{\text { Total number of interactions attempted by the } i \text {-th agent }} .
$$

The rationale of this assumption being the fact that an agent with many successful interactions will be more motivated to wait shorter times between one attempt and the next one than a peer with an history of many failed attempts. Our assumption is that the latter will have a higher probability to wait longer than the former before finding the "courage" of trying to approach someone else.

\footnotetext{
${ }^{3}$ An agent engaged in a discussion will be defined to be occupied, while an agent waiting for an available partner or proposing a conversation will be defined to be free.
} 


\subsection{Opinion exchange (Model B)}

The alternative hypothesis is that scientists interact, in the context of scientific conferences, in order to exchange opinions, for instance to try to convince their peers of some idea. In order to test this conjecture, we slightly modify the previous model by introducing the opinion exchange in the form proposed by [6] (this second model is hereby named Model B). The main novelty of this second setup is that all agents are also endowed with an opinion $\left(O_{i}\right)_{1 \leq i \leq N}$, initially uniformly distributed in the interval $[0,1]$, and that this opinion has a feedback on the dynamics of the model. More

precisely we assume that the distribution of the interaction times, $\frac{1}{(\Delta t)^{\gamma}}$, depends on the opinions difference among the two interacting agents: $\gamma=1.5+\left|O_{i}-O_{j}\right|$. The rationale being that the more affine the agents are, the higher is the probability their conversation will last longer: having similar opinions, the two agents can build a common ground and continue longer the discussion.

In line with [6], the social interaction can produce an opinions update if the agents are not too far in the opinion space, more precisely once agents $i$ and $j$ meet if $\left|O_{i}-O_{j}\right| \leq \sigma$, being $\sigma \in(0,1)$ a proxy for the openness of mind, their opinions will tend to converge:

$$
\begin{gathered}
O_{i}^{t+\Delta t}=O_{i}^{t}+\mu(\Delta t)\left(O_{j}^{t}-O_{i}^{t}\right) \\
O_{j}^{t+\Delta t}=O_{j}^{t}+\mu(\Delta t)\left(O_{i}^{t}-O_{j}^{t}\right) .
\end{gathered}
$$

We assumed that the convergence parameter, $\mu$, depends on the duration of the discussion:

$$
\mu(\Delta t)=\frac{1}{2} \tanh (\beta \Delta t),
$$

this results in a effectiveness of the conversation: the longer lasts the interaction, i.e. the greater $\Delta t$, the stronger will be the convergence of the opinions to their average. This choice is made to mimic the fact that during a long conversation one has comparatively more chances of changing idea, or to convince the partner, than during a short chat. 


\section{The results}

We simulate both models for a time duration of $T=12$ hours, once again to compare our results with [4]. At the end of the simulations, each agent is characterized by the number of distinct contacts he had, that can be called the degree $k_{i}$ of the interaction network, and by the total discussion time he had, namely the strength $s_{i}$. The latter relates to the total discussion time, $w_{i j}$ (weight), agents $i$ and $j$ had, by $s_{i}=\sum^{\prime} w_{i j}$, being the sum restricted to agents $j$ that had at least a contact with $i$.

In this preliminary work we focused on the relationship between the degree $k$ and the average node strength $\langle s(k)>$ as a function of the degree:

$$
<s(k)>=\frac{\sum_{i: k_{i}=k} s_{i}}{N_{k}},
$$

where $N_{k}$ denotes the total number of agents with degree $k$.
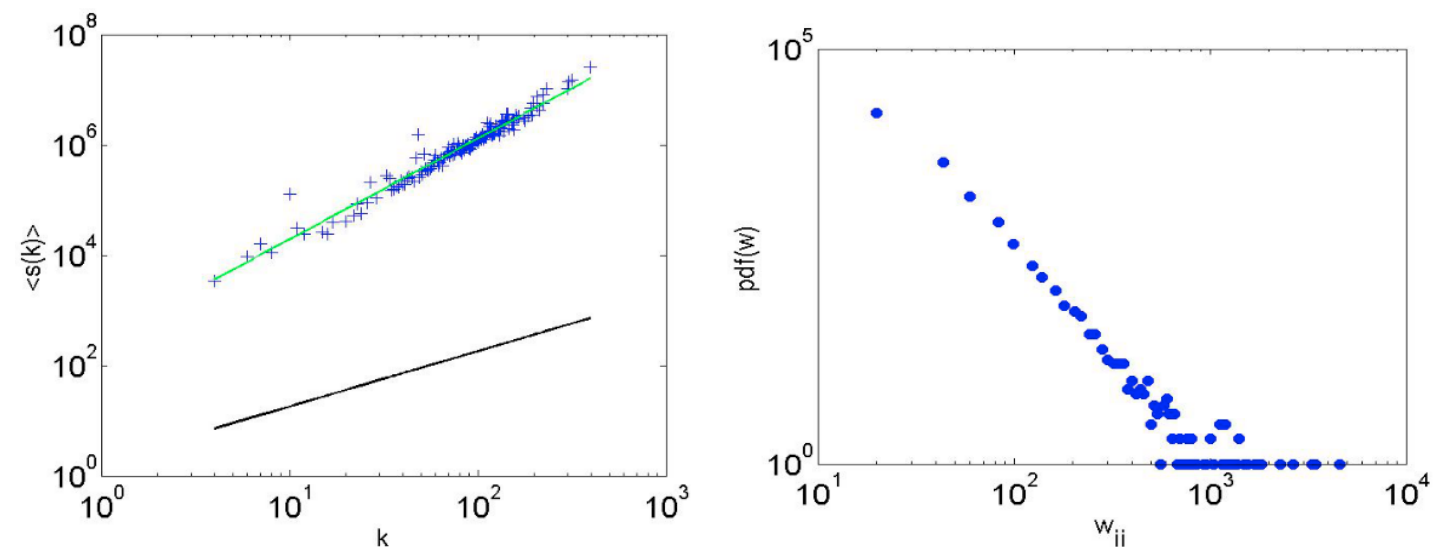

Fig. 1. Average strength versus degree and weight distribution (Model A). On the left panel, the log-log plot of the average strength for a generic simulation with: $N=575$ and $\gamma_{h}=3.0$, together with a best linear fit (lighter line), the black line denotes the linear growth. On the right panel, the probability distribution function of agents weights for the same simulation. 
As it can be observed from Figure 1 a generic simulation of our model generates a pretty well defined super-linear relationship between the degree and the average strength. The data extracted from Barrat et al. [4] show that the relationship between the degree and the average node strength is given by $<s(k)>\sim k^{1.73}$. We are able to tune the model parameters, in particular $\gamma_{h}$ responsible for the group organization, in order to obtain results which are significantly close to those obtained experimentally. Moreover the matching between the fit on empirical data and the synthetic ones obtained via our models, allows us to gain insights into which kind of activity is dominant in a certain dataset.

Both models are, in principle, capable of generating outcomes similar to the experimental ones; however our aim is to give a social interpretation to the parameters values, allowing us to unravel the mechanism at play : which is the social force, networking or opinion exchange, responsible for the measured observables, average strength? In particular we are interested in studying different scale free distributions of agents reputation. We observe that, in a group where agents with very high reputation are present, people tend to do networking, in fact the Model A generates a slope much closer to the experimental one than the Model B. On the contrary when the group of interacting agents is constituted mainly by equally reputed agents, the Model B seems to fit better the experimental data. These results are summarized in Table 1, for a given set of parameters values.

\begin{tabular}{|c|c|c|}
\hline$\rho$ & $\begin{array}{c}\text { Model A } \\
\text { (Networking) }\end{array}$ & $\begin{array}{c}\text { Model B } \\
\text { (Opinion Exchange) }\end{array}$ \\
\hline$\gamma_{h}=3$ & $\sim 1.8$ & $\sim 1.6$ \\
\hline$\gamma_{h}=1.1$ & $\sim 1.6$ & $\sim 1.8$ \\
\hline
\end{tabular}

Table 1. Super-linear growth of the average strength vs the degree $:\left\langle s(k)>\sim k^{\rho}\right.$, for different values of $\gamma_{h}$ for Model A and Model B. 


\section{Extension: Mixed population hypothesis}

In the analyses performed in the previous section we showed that it is possible to fit the data observed by [4] with two different models. Both are based on the assumption that each member of the observed population share the same objective (networking or opinion exchange).

It could be argued that, in reality, not every participant to a conference has the same objective. Some people may be willing to share and possibly change opinions, while others may just want to do networking.

In order to make our model more realistic we now relax the homogeneity assumption of group's objective, allowing for a mixed population, where $p N$ agents are willing to discuss while $(1-p) N$ agents do networking. The similar structure of Models A and B makes the task of merging them relatively straightforward. When two agents of the same type are selected to initiate an interaction we can simply assume that the same rules outlined in Section 1, for the homogenous population case, apply. The only complication comes when two agents of different types meet. In this case there are two conflicting rules for the extraction of the interaction time. To solve this problem we observe that a conversation can last only as long as both the participants are willing to pursue it. Thus, we assume that each of the agents extracts an interaction time corresponding to his own rule of behavior (a networker

as in model $\mathrm{A}$ and an opinion exchanger as in model B) and the actual length of the conversation is the shortest of the two. Moreover, the agent interested in exchanging opinions, may change his own idea while the agent, only interested in knowing someone more important, will keep the same opinion as before.

With this unified modeling setup, we are able to study the effect of the population' composition on the relationship between agents' connectivity and the average strength of their interactions, $\left\langle s(k)>\sim k^{\rho}\right.$. In Figure 2 we show the dependence of 
the exponent $\rho$ on the fraction, $p$, of agents interested into discussing, for different values of the parameters $\gamma, \gamma_{h}$ and $\gamma_{w}$.
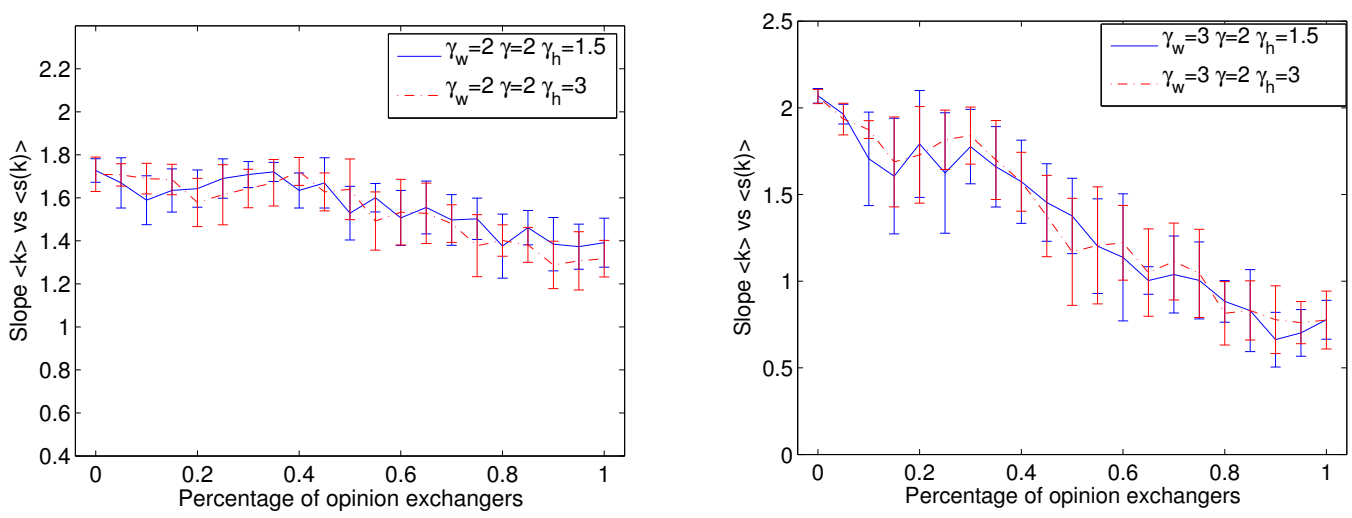

Fig. 2. Dependence of $\rho$ on $p$, where $<s(k)>\sim k^{\rho}$ and $p$ is the fraction of agents aiming at exchanging opinion. On the left panel, we report numerical results for the set of parameters: $N=575, \gamma=2, \gamma_{w}=2$ and $\gamma_{h}=\{1.5,3.0\}$, while on the right panel: $N=575, \gamma=2, \gamma_{w}=3$ and $\gamma_{h}=\{1.5,3.0\}$.

One can immediately observe that the distribution of h-indexes in the population (driven by $\gamma_{h}$ ) does not have a large influence on $\rho$. Moreover, from the data one can also deduce that $p$ should be larger enough, i.e. larger than 0.4 , to have an appreciable influence on the value of $\rho$, for instance to make it to decrease. Finally, this trend is stronger the larger is $\gamma_{w}$ (i.e. the less likely is for an agent to wait for long times). We propose the following explanation for this phenomenon. When $\gamma_{w}$ is small, agents are more likely to wait for longer amounts of time after a failed interaction and thus they can be considered available once a second free agent tries to contact them. [vedi se ho capito bene quello che volevi dire, perche' mi sembra in contradizione il fatto che aspettino molto e che al tempo stesso siano liberi]. Whenever a large amount of persons are not engaged in conversations, there is an higher probability to have positive interactions (and thus less "frustration"). The consequence is that 
the presence of a significative number of opinion exchangers increases the length of interactions between them thus leading to lower $\rho$. When $\gamma_{w}$ increases the coefficient $\rho$ decreases even more because the effect of the introduction of opinion exchanges on their relative interactions is stronger.

\section{Conclusions}

In this paper we presented and studied a simple Agent Based Model, grounded on experimental data, with the aim of reproducing some macroscopic measured features, for instance the super linear growth of the total discussion times as a function of the number of distinct contacts agents had. Moreover we are able to provide a sociological interpretation of the parameters values allowing us to make a distinction between the case where individuals engage discussions with a networking goal, with respect to the case where individuals meet to exchange opinions and try to convince, or to be convoked [cosa vuoi dire?], by their partners.

The model is constructed upon quite simple social interaction rules and strongly use some experimentally measured data, notably the distribution of contact times. The model is voluntarily simple, relying on few parameters, whose role can be completely understood, and thus to be able to provide a social interpretation of the latter.

We agree that the model could be improved by allowing several discussion topics, while in the present form only one subject is at play. This modification could be easily introduced in a forthcoming paper, however we stress that even under the proposed very simple scenario, the model is able to reproduce some real features according to the social structure of the group. We believe that this fact can be ascribed to the use of measured data that contain important hidden informations about the individuals and their actions, that otherwise could be very difficulty modeled and/or need the introduction of several unknown parameters. For instance, in the reality agents are not homogeneous and each one has his own "discussion strategy" or his 
own "use of time strategy" that are very difficult to evaluate; however this information is captured by the distribution of contacts times and thus we avoid the introduction of a complicated modeling for each agent.

We believe that such strategy, where models are built using simple social rules together with experimental data, could be very fruitful in the future to gain new insights into social dynamics.

\section{References}

1. Axelrod R. (1997) The dissemination of Culture: A model with local convergence and global polarization, The Journal of Conflict resolution, Vol. 41 (2), pp. 203.

2. Blondel, V et al. (2010) Regions and borders of mobile telephony in Belgium and around Brussels, Brussels Studies, Vol. 42.

3. Blondel V, (2008) Fast unfolding of communities in large networks, Journal of Statistical Mechanics, pp. 1742-5468, P10008.

4. Cattuto C et al. (2010) Dynamics of Person-to-Person Interactions from Distributed RFID Sensor Networks. PLoS ONE, Vol. 5(7), pp. e11596.

5. Chamberlin, E.H. (1948) An experimental imperfect market, J.Pol. Econ., Vol. 56, pp. 95.

6. Deffuant G. et al. (2000) Mixing beliefs among interacting agents, Advances in Complex Systems, Vol. 3, pp. 87-98.

7. Galam S. (1986) Majority rule, Hierarchical structures and democratic totalitarism: A statistical approach, Journal of Mathematical Psychology, Vol. 30, pp. 426.

8. Gerard H.G. and Orive R. (1987) The dynamics of opinion formation, Advances in experimental social psychology, Vol. 20, pp. 171

9. Krings G. et al., (2009) Urban gravity: a model for inter-city telecommunication flows, Journal of Statistical Mechanics, pp. L07003.

10. Isella, L et al. (2011) What's in a crowd? Analysis of face-to-face behavioral networks, Journal of Theoretical Biology, Vol. 271, pp. 166.

11. Lambiotte R. et al. (2008) Geographical dispersal of mobile communication networks. Physica A, Vol. 387, pp. 5317-5325.

12. Onella, J.P et al (2007) Analysis of large-scale weighted network of one-to-one human communication, New journal of physics, Vol. 179.

13. Weisbuch, G., et al. (2002) Meet, discuss and segregate! , Complexity, Vol. 7, pp. 55-63.

14. Schelling T.C. (1971) Dynamic models of Segregation, Journal of Mathematical Sociology, Vol. 1, pp. 143.

15. Sonnemans J. van Dijk F. and van Winden F. (2006) On the dynamics of social ties structures in groups, Journal of Personality and Social Psychology, Vol. 43 (1), pp. 78.

16. Zhao et al (2011) Social network dynamics of face to face interactions, Physical Review E, Vol. 83, pp. 056109. 